\title{
Additive and multiplicative effects network models
}

\author{
Peter D. Hoff \\ Department of Statistical Science \\ Duke University
}

July 24, 2018

\begin{abstract}
Network datasets typically exhibit certain types of statistical dependencies, such as withindyad correlation, row and column heterogeneity, and third-order dependence patterns such as transitivity and clustering. The first two of these can be well-represented statistically with a social relations model, a type of additive random effects model originally developed for continuous dyadic data. Third-order patterns can be represented with multiplicative random effects models, which are related to matrix decompositions commonly used for matrix-variate data analysis. Additionally, these multiplicative random effects models generalize other popular latent variable network models, such as the stochastic blockmodel and the latent space model. In this article we review a general regression framework for the analysis of network data that combines these two types of random effects and accommodates a variety of network data types, including continuous, binary and ordinal network relations.

Keywords: Bayesian, factor model, generalized linear model, latent variable, matrix decomposition, mixed effects model.
\end{abstract}

\section{Introduction}

Network data provide quantitative information about relationships among objects, individuals or entities, which we refer to as nodes. Most network data quantify pairwise relationships between nodes. A pair of nodes is referred to as a dyad, and a quantity that is measured or observed for multiple dyads is called a dyadic variable. Common sample spaces for dyadic variables include continuous, discrete, dichotomous and ordinal spaces, among others. Examples of dyadic variables 
include quantitative measures of trade flows between countries, communications among people, binding activity among proteins, and structural connections among regions of the brain, to name just a few.

Measurements of a dyadic variable on a population of $n$ nodes may be summarized with a sociomatrix, an $n \times n$ square matrix $\mathbf{Y}$ with an undefined diagonal, where entry $y_{i, j}$ denotes the value of the relationship between nodes $i$ and $j$ from the perspective of node $i$, or in the direction from $i$ to $j$. Analysis of an observed sociomatrix $\mathbf{Y}$ often proceeds in the context of one or more statistical models, with which a data analyst may evaluate competing theories of network formation, describe patterns in the network, estimate effects of other variables on dyadic relations, or impute missing values.

While most of the dyadic variables I have encountered are not dichotomous in their raw form, much of the statistical literature has focused on binary network data for which the sociomatrix $\mathbf{Y}$ can be viewed as the adjacency matrix of a graph. Many statistical random graph models are motivated by intuitive, preconceived notions of how networks may form, particularly social networks. For example, preferential attachment models view an observed network as the end result of a social process in which nodes are sequentially introduced into a population of existing nodes (Price, 1976). As another example, the parameters in the types of exponential family graph models that are commonly used have interpretations as node-level preferences for certain relationship outcomes (Wasserman and Pattison, 1996).

An alternative approach is to build a statistical model for $\mathbf{Y}$ based on its inherent structure as a sociomatrix, that is, as a data matrix whose row labels are the same as its column labels. Such an approach can build upon familiar, well-developed statistical methodologies such as ANOVA, linear regression, matrix decompositions, factor analysis and linear and generalized linear mixed effects models, and can be applied to a wide variety of dyadic data types. In this article, we review such a framework for network data analysis using these tools, starting with simple ANOVAstyle decompositions of sociomatrices and ending with additive and multiplicative random effects regression models for continuous, binary, ordinal and other types of dyadic network data.

In the next section we review an ANOVA-style decomposition of a sociomatrix known as the social relations model (SRM) (Warner et al., 1979, Wong, 1982), which corresponds to a particular Gaussian additive random effects model for network data. An extension of this model that includes 
covariates is also developed, which we call the social relations regression model (SRRM). The SRM and SRRM are able to describe network variances and covariances, but are unable to describe third-order dependence patterns such as transitivity, balance, or the existence of clusters of nodes with high subgroup densities of ties. In Section 3 we discuss how such patterns can be represented by a multiplicative latent factor model, in which the relationship between two nodes depends on the similarity of their unobserved latent factors. From a matrix decomposition perspective, this motivates the use of an "additive main effects, multiplicative interaction" (AMMI) matrix model Gollob, 1968, Bradu and Gabriel, 1974). Combining an AMMI model with a social relations covariance model yields what we call an additive and multiplicative effects (AME) network model.

These AME models are built from linear regression, random effects models and matrix decomposition - methods which are most appropriate for continuous data consisting of a signal of interest plus Gaussian noise. In contrast, many dyadic variables are discrete, ordinal, binary or sparse. In Section 4 we extend the AME framework to accommodate these and other types of dyadic variables using a Gaussian transformation model. In Section 5 we compare the multiplicative effects component of an AME model with two other latent variable network models, the stochastic blockmodel (Nowicki and Snijders, 2001) and the latent space model (Hoff et al., 2002). We review results showing that these latter two models can be viewed as submodels of the multiplicative effects model. Connections to exponentially parameterized random graph models (ERGMs) (Wasserman and Pattison, 1996) are also discussed. Section 6 presents a Markov chain Monte Carlo algorithm for Bayesian model fitting of a hierarchy of AME network models. A discussion follows in Section 7.

\section{Social Relations Regression}

\subsection{ANOVA and the Social Relations Model}

Numeric sociomatrices typically exhibit certain statistical features. For example, it is often the case that values of the dyadic variable in a given row of the sociomatrix are correlated with one another, in the sense that high and low values are not equally distributed among the rows, resulting in substantial heterogeneity of the row means of the sociomatrix. Such heterogeneity can be explained by the fact that the relations within a row all share a common "sender," or row index. If sender $i_{1}$ 
is more "sociable" than sender $i_{2}$, we would expect the values in row $i_{1}$ to be larger than those in row $i_{2}$, on average. In this way, heterogeneity of the nodes in terms of their sociability contributes to an across-row variance of the row means of the sociomatrix. Similarly, nodal heterogeneity in "popularity" contributes to the across-column variance of the column means.

A classical approach to evaluating across-row and across-column heterogeneity in a data matrix is the ANOVA decomposition. A statistical model based on the ANOVA decomposition posits that the variability of the $y_{i, j}$ 's around some overall mean $\mu$ is well-represented by additive row and column effects:

$$
y_{i, j}=\mu+a_{i}+b_{j}+\epsilon_{i, j}
$$

In this model, heterogeneity among the $a_{i}$ 's and $b_{j}$ 's gives rise to observed heterogeneity in the row means and column means of the sociomatrix, respectively.

While straightforward to implement, a classical ANOVA analysis ignores a fundamental characteristic of dyadic data: Each node appears in the dataset as both a sender and a receiver of relations, or equivalently, the row and column labels of the data matrix refer to the same set of nodes. In the context of the ANOVA model, this means that each node $i$ has two additive effects: a row effect $a_{i}$ and a column effect $b_{i}$. Since each pair of effects $\left(a_{i}, b_{i}\right)$ shares a node, a correlation between the vectors $\left(a_{1}, \ldots, a_{n}\right)$ and $\left(b_{1}, \ldots, b_{n}\right)$ may be expected. Additionally, each dyad $\{i, j\}$ has two outcomes, $y_{i, j}$ and $y_{j, i}$. As such, the possibility that $\epsilon_{i, j}$ and $\epsilon_{j, i}$ are correlated should be considered.

We illustrate these phenomena empirically with a sociomatrix of export data among $n=30$ countries. Here, $y_{i, j}$ is the 1990 export volume from country $i$ to country $j$, in log billions of dollars. For each country $i=1, \ldots, n, \hat{a}_{i}$ the $i$ th row mean minus the grand mean $\hat{\mu}$ of the sociomatrix, and $\hat{b}_{i}$ is the $i$ th column mean minus $\hat{\mu}$. The left panel of Figure 1 shows that these row and column effects are strongly correlated - countries with large export volumes typically have larger than average import volumes as well. A scatterplot of $\hat{\epsilon}_{i, j}=y_{i, j}-\left(\hat{\mu}+\hat{a}_{i}+\hat{b}_{j}\right)$ versus $\hat{\epsilon}_{j, i}$ in the right panel of the plot indicates a strong dyadic correlation, even after controlling for country-specific heterogeneity in export and import volumes.

The standard ANOVA model of a data matrix quantifies row variation, column variation and residual variation. However, the ANOVA model does not quantify the sender-receiver or dyadic correlations that are apparent from the figure, and that are present in most other dyadic datasets I 

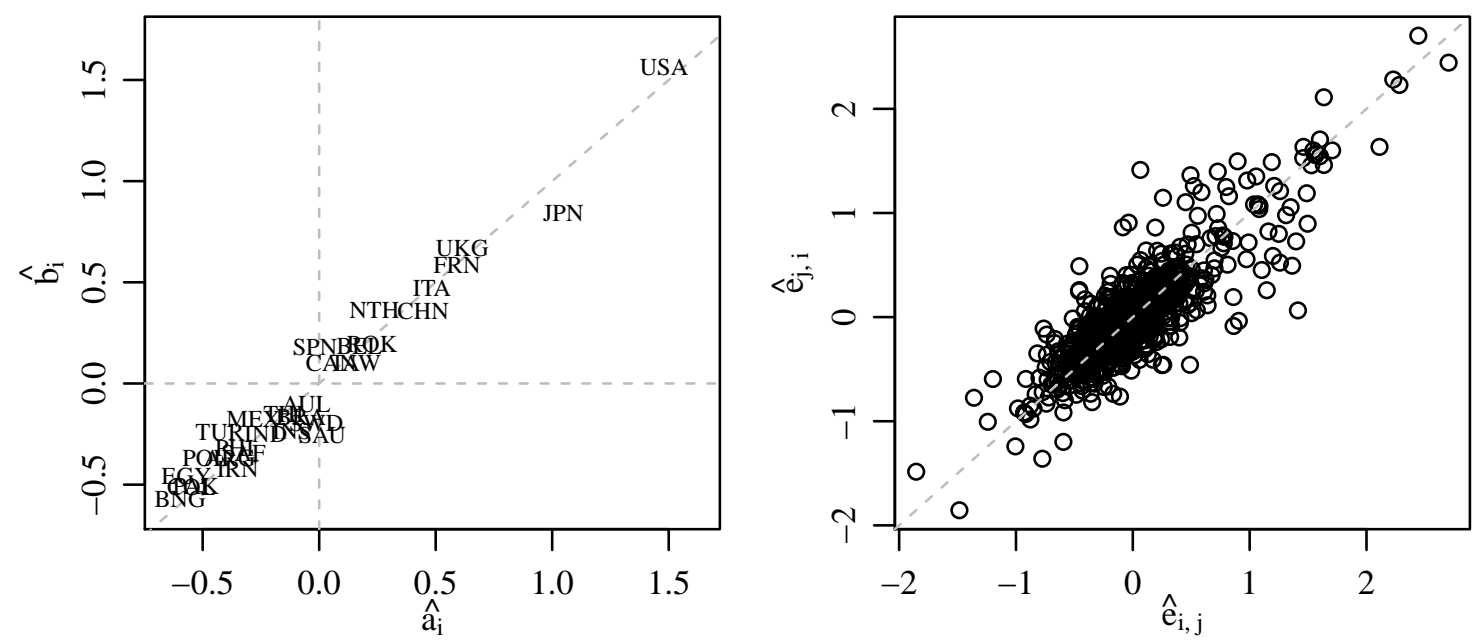

Figure 1: Left panel: Scatterplot of country-level export effects versus import effects. Right panel: Scatterplot of dyadic residuals.

have seen. A model that does quantify these correlations, and therefore provides a more complete description of the sociomatrix, was introduced in the psychometrics literature by Warner et al. (1979). This more complete model, called the social relations model (SRM), is a random effects model given by 2.1 but with the additional assumptions that

$$
\operatorname{Var}\left[\left(\begin{array}{c}
a_{i} \\
b_{i}
\end{array}\right)\right]=\Sigma=\left(\begin{array}{cc}
\sigma_{a}^{2} & \sigma_{a b} \\
\sigma_{a b} & \sigma_{b}^{2}
\end{array}\right) \quad \operatorname{Var}\left[\left(\begin{array}{c}
\epsilon_{i, j} \\
\epsilon_{j, i}
\end{array}\right)\right]=\sigma^{2}\left(\begin{array}{cc}
1 & \rho \\
\rho & 1
\end{array}\right),
$$

with effects otherwise being independent. Straightforward calculations show that under this random effects model, the variance of the relational variable is $\operatorname{Var}\left[y_{i, j}\right]=\sigma_{a}^{2}+2 \sigma_{a b}+\sigma_{b}^{2}+\sigma^{2}$, and the covariances among the relations are

$$
\begin{array}{rlrl}
\operatorname{Cov}\left[y_{i, j}, y_{i, k}\right] & =\sigma_{a}^{2} & \text { (within-row covariance) } \\
\operatorname{Cov}\left[y_{i, j}, y_{k, j}\right] & =\sigma_{b}^{2} & & \text { (within-column covariance) } \\
\operatorname{Cov}\left[y_{i, j}, y_{j, k}\right] & =\sigma_{a b} & \text { (row-column covariance) } \\
\operatorname{Cov}\left[y_{i, j}, y_{j, i}\right] & =2 \sigma_{a b}+\rho \sigma^{2} & \text { (row-column covariance plus reciprocity) }
\end{array}
$$

with all other covariances between elements of $\mathbf{Y}$ being zero. We refer to this covariance model as the social relations covariance model. Unbiased moment-based estimators of $\mu, \Sigma, \sigma^{2}$ and $\rho$ are derived in Warner et al. (1979), and standard errors for these estimators are obtained in Bond 


\begin{tabular}{r|ccc|ccc|ccc}
\hline & \multicolumn{3}{|c|}{ IID } & \multicolumn{3}{c}{ SRRM } & \multicolumn{3}{c}{ AME } \\
\hline regressor & $\hat{\beta}$ & $\operatorname{se}(\hat{\beta})$ & $t$-ratio & $\hat{\beta}$ & $\operatorname{se}(\hat{\beta})$ & $t$-ratio & $\hat{\beta}$ & $\operatorname{se}(\hat{\beta})$ & $t$-ratio \\
\hline exporter polity & 0.015 & 0.004 & 4.166 & 0.015 & 0.016 & 0.934 & 0.012 & 0.016 & 0.782 \\
importer polity & 0.022 & 0.004 & 6.070 & 0.022 & 0.016 & 1.419 & 0.018 & 0.015 & 1.190 \\
exporter GDP & 0.411 & 0.021 & 19.623 & 0.407 & 0.095 & 4.302 & 0.346 & 0.103 & 3.373 \\
importer GDP & 0.398 & 0.020 & 19.504 & 0.397 & 0.094 & 4.219 & 0.336 & 0.103 & 3.250 \\
distance & -0.057 & 0.004 & -13.360 & -0.064 & 0.005 & -11.704 & -0.041 & 0.004 & -10.970
\end{tabular}

Table 1: Parameter estimates and standard errors from the trade data using a normal linear regression model with i.i.d. errors, a SRRM, and an AME model.

and Lashley (1996). Under the additional assumption that the random effects are jointly normally distributed, Wong (1982) provides an EM algorithm for maximum likelihood estimation, Gill and Swartz (2001) develop a Bayesian method for parameter estimation, and Li and Loken (2002) discuss connections to models in genetics and extensions to repeated-measures dyadic data.

\subsection{Social relations regression models}

Often we wish to quantify the association between a particular dyadic variable and some other dyadic or nodal variables. Useful for such situations is a type of linear mixed effects model we refer to as the social relations regression model (SRRM), which combines a linear regression model with the covariance structure of the SRM as follows:

$$
y_{i, j}=\boldsymbol{\beta}^{\top} \mathbf{x}_{i, j}+a_{i}+b_{j}+\epsilon_{i, j},
$$

where $\mathbf{x}_{i, j}$ is a $p$-dimensional vector of regressors and $\boldsymbol{\beta}$ is a vector of regression coefficients to be estimated. The vector $\mathbf{x}_{i, j}$ may contain variables that are specific to nodes or pairs of nodes. For example, we may have $\mathbf{x}_{i, j}=\left(\mathbf{x}_{r, i}, \mathbf{x}_{c, j}, \mathbf{x}_{d, i, j}\right)$ where $\mathbf{x}_{r, i}$ is a vector of characteristics of node $i$ as a sender or row object, $\mathbf{x}_{c, j}$ is a vector of characteristics of node $j$ as a receiver or column object, and $\mathbf{x}_{d, i, j}$ is a vector of characteristics of the ordered pair $(i, j)$.

We illustrate the use of the SRRM with a more detailed analysis of the international trade dataset described above. This dataset also includes several other variables, such as country-specific measures of gross domestic product (GDP) and polity (a measure of citizen access to government), as well as the geographic distance between pairs of county capitals. Our objective in this example 
is to quantify the relationship between trade and polity after controlling for the effects of GDP and geographic distance. We first do so with a naive ordinary linear regression model of the form

$$
y_{i, j}=\beta_{0}+\beta_{r, 1} \text { polity }_{i}+\beta_{r, 2} \operatorname{gdp}_{i}+\beta_{c, 1} \text { polity }_{j}+\beta_{c, 2} \operatorname{gdp}_{j}+\beta_{d} \text { distance }_{i, j}+\epsilon_{i, j},
$$

where polity $_{i}$ is a measure of country $i$ 's polity score on a scale from 1 to $10, \operatorname{gdp}_{i}$ is the log GDP of country $i$ in dollars, distance $e_{i, j}$ is the log distance in miles between capitals of countries $i$ and $j$, and the $\epsilon_{i, j}$ 's are assumed to be i.i.d. mean-zero error terms. This model is a "gravity model"

of trade (Isard, 1954, Bergstrand, 1985), where trade flow is analogous to a gravitational force between countries, and GDP plays the role of mass. Gravity models of this type are widely used to empirically evaluate different theories of international trade (Baier and Bergstrand, 2009).

Regression parameter estimates and standard errors assuming an i.i.d. error model are given in the first column of Table 1. Based upon the ratio of parameter estimates to standard errors, we would conclude that the hypothesis of no polity effects is inconsistent with an i.i.d. error model. However, while technically valid, this conclusion is not particularly interesting given that we expect row, column and dyadic dependence for network data such as these, and thus doubt the i.i.d. error model a priori. More interesting is an evaluation of whether or not the hypothesis of no polity effects is consistent with a social relations covariance model. The parameter estimates and standard errors for the SRRM in the second column of the table indicate that indeed it is: the parameter estimates of the polity effects are not substantially larger than their standard errors.

\section{Multiplicative Effects Models}

While more reasonable than an ordinary regression model, SRRMs applied to many datasets often exhibit substantial lack of fit. In particular, it is often observed that real networks exhibit patterns of dependence among triples of nodes such as transitivity, balance and clustering (Wasserman and Faust, 1994). For example, in the context of fitting a regression model, the notion of balance would correspond to there generally being a higher-than expected relationship (i.e. a positive residual) between nodes $j$ and $k$ if that between $i$ and $j$ and $i$ and $k$ were both also higher than expected. Such patterns can be quantified with summary statistics such as $\sum_{i, j, k} \hat{\epsilon}_{i, j} \hat{\epsilon}_{j, k} \hat{\epsilon}_{k, i}$, where $\hat{\epsilon}_{i, j}$ is a residual from a least-squares fit. Figure 2 displays the posterior predictive distribution of this statistic from a Bayesian fit of the SRRM to the trade data. The predictive distribution of this 


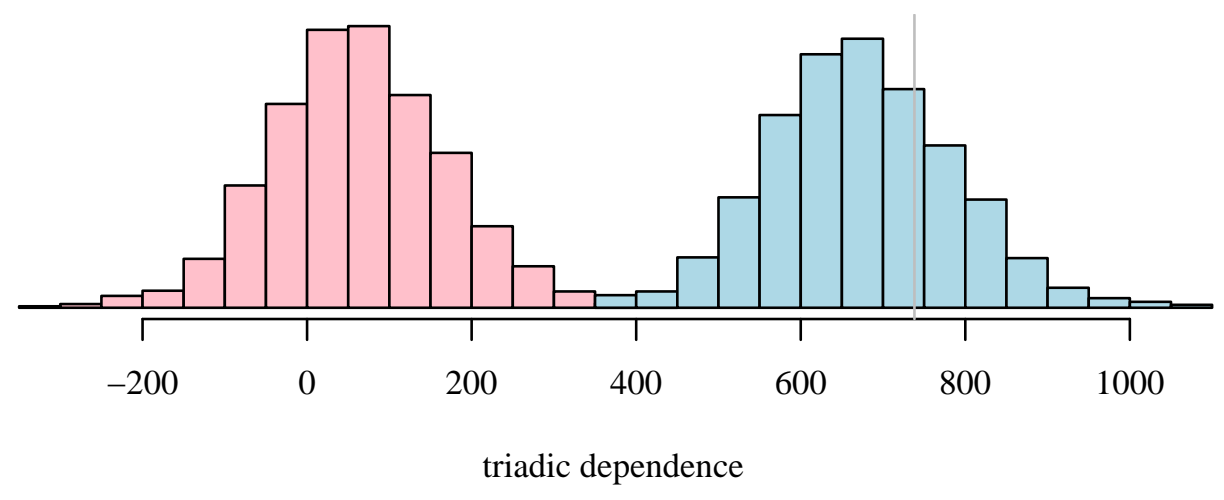

Figure 2: Posterior predictive distributions of a triadic goodness of fit statistic. The pink histogram corresponds to the SRRM fit, the blue to the AME fit. The observed value of the statistic is given by the vertical gray line.

statistic under the SRRM does not overlap with the observed value, indicating that the SRRM is inconsistent with this feature of the data.

The SRRM, or any other Gaussian random effects model, is unable to describe a third-order dependence pattern such as this because all third-order moments of mean-zero Gaussian random variables are zero. To model such patterns we must move beyond linear Gaussian random effects models where random effects and error terms combine additively. One solution is to consider additional random effects that combine nonadditively. For example, let $\gamma_{i, j}=\mathbf{u}_{i}^{\top} \mathbf{v}_{j}$, where $\mathbf{u}_{i}$ and $\mathbf{v}_{i}$ are $r$-dimensional mean-zero latent Gaussian vectors, i.i.d. across nodes with $\operatorname{Cov}\left[\mathbf{u}_{i}, \mathbf{v}_{i}\right]=\Psi_{u v}$. Then

$$
\begin{aligned}
\mathrm{E}\left[\gamma_{i, j} \gamma_{j, k} \gamma_{k, i}\right] & =\mathrm{E}\left[\mathbf{u}_{i}^{\top} \mathbf{v}_{j} \mathbf{u}_{j}^{\top} \mathbf{v}_{k} \mathbf{u}_{k}^{\top} \mathbf{v}_{i}\right] \\
& =\mathrm{E}\left[\mathbf{u}_{i}^{\top} \mathbf{v}_{i}\right]^{3}=\operatorname{tr}\left(\Psi_{u v}\right)^{3} .
\end{aligned}
$$

Therefore, a social relations regression model that includes second- and third-order residual depen- 
dencies is given by

$$
\begin{aligned}
y_{i, j} & =\boldsymbol{\beta}^{\top} \mathbf{x}_{i, j}+\mathbf{u}_{i}^{\top} \mathbf{v}_{j}+a_{i}+b_{j}+\epsilon_{i, j} \\
\left(\mathbf{u}_{1}, \mathbf{v}_{1}\right), \ldots,\left(\mathbf{u}_{n}, \mathbf{v}_{n}\right) & \sim \text { i.i.d. } N_{2 r}(\mathbf{0}, \Psi) \\
\left(a_{1}, b_{1}\right), \ldots,\left(a_{n}, b_{n}\right) & \sim \text { i.i.d. } N_{2}(\mathbf{0}, \Sigma) \\
\left\{\left(\epsilon_{i, j}, \epsilon_{j, i}\right): i<j\right\} & \sim \text { i.i.d. } N_{2}\left(\mathbf{0}, \sigma^{2}\left(\begin{array}{ll}
1 & \rho \\
\rho & 1
\end{array}\right)\right) .
\end{aligned}
$$

We call such a model an additive and multiplicative effects model (AME). Specifically, we refer to the model given by (3.1) as a Gaussian AME, since the observed data are conditionally Gaussian, given $\boldsymbol{\beta}$ and the multiplicative effects. A rudimentary multiplicative effects model appeared in Hoff et al. (2002), along with some other nonadditive random effects models. A symmetric multiplicative effects model was combined with the social relations covariance model in Hoff (2005), and versions of (3.1) were studied and developed in Hoff (2008, 2009) and Hoff et al. (2013).

The matrix form of this model can be expressed as

$$
\mathbf{Y}=\mathbf{M}+\mathbf{a} \mathbf{1}^{\top}+\mathbf{1} \mathbf{b}^{\top}+\mathbf{U} \mathbf{V}^{\top}+\mathbf{E}
$$

where $m_{i, j}=\boldsymbol{\beta}^{\top} \mathbf{x}_{i, j}, \mathbf{a}=\left(a_{1}, \ldots, a_{n}\right), \mathbf{b}=\left(b_{1}, \ldots, b_{n}\right)$ and $\mathbf{U}$ and $\mathbf{V}$ are $n \times r$ matrices with $i$ th rows equal to $\mathbf{u}_{i}$ and $\mathbf{v}_{i}$ respectively, with $r$ being the length of each of these latent vectors. This represents the deviations of $\mathbf{Y}$ from the linear regression model $\mathbf{M}$ as the sum of a rank-1 matrix of row effects, a rank-1 matrix of column effects, a rank- $r$ matrix $\mathbf{U V}^{\top}$ and a noise matrix $\mathbf{E}$. Absent a covariance model for the node-specific effects or dyadic residuals, this representation is essentially a special case of an additive main effects, multiplicative interaction (AMMI) model (Gollob, 1968, Bradu and Gabriel, 1974), a class of matrix models developed in the psychometric and agronomy literature for data arising from two-way layouts with no replication. Since sociomatrices have additional structure - the row factors are the same as the column factors - our random effects version of the AMMI model includes the SRM covariance model for the $a_{i}$ 's, $b_{i}$ 's and $\epsilon_{i, j}$ 's, in addition to a random effects model for $\mathbf{u}_{i}$ and $\mathbf{v}_{i}$ to represent possible third-order dependencies in the sociomatrix. We refer to this model as an additive and multiplicative effects model, or AME model for dyadic network data.

To illustrate the how the inclusion of multiplicative effects improves model fit, we obtain the posterior predictive distribution of the triadic goodness-of-fit statistic $\sum_{i, j, k} \hat{\epsilon}_{i, j} \hat{\epsilon}_{j, k} \hat{\epsilon}_{k, i}$ under an 

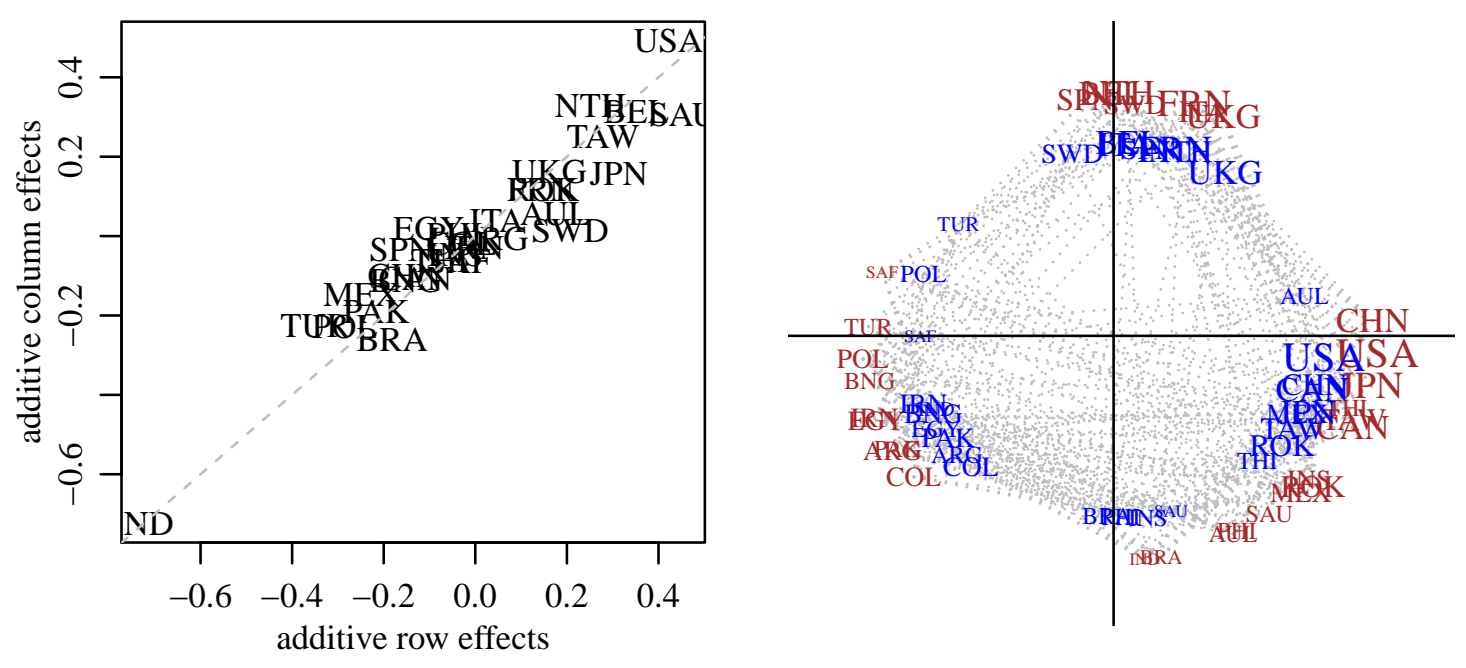

Figure 3: Estimates of node-specific effects. The left panel gives additive row effects versus additive column effects. The plot on the right gives estimates of $\mathbf{u}_{i}$ in red and $\mathbf{v}_{i}$ in blue for each country $i=1, \ldots, n$. The country names indicate the direction of these vectors, and the size of the plotting text indicates their magnitude. A dashed line is drawn between an export-import pair if their trade flow is larger than expected based on the other terms in the model.

AME model with two-dimensional multiplicative effects and the same regressors as the SRRM (polity, GDP and geographic distance). A histogram of this posterior predictive distribution is given in Figure 2, along with that of the SRRM fit. The posterior predictive distribution obtained under the AME fit is roughly centered around the observed value of the statistic indicating that, unlike the SRRM, the AME model is able to describe this third-order residual dependency in the trade data. Finally, parameter estimates and standard errors for the regression coefficients in this AME model are given in the third column of Table 1. Parameter estimates are slightly smaller than those of the SRRM, but the main conclusions remain the same.

From a random effects perspective, the multiplicative effect $\mathbf{u}_{i}^{\top} \mathbf{v}_{j}$ can be viewed as a means to quantify third order dependence. However, these effects can also be interpreted as representing omitted regression variables or uncovering group structure among the nodes. This interpretation is based on the observation that the strength or presence of ties between nodes is often related to similarities of node-level attributes. For example, suppose for each node $i$ that $x_{i}$ is the indicator that $i$ is a member of a particular group or has a particular trait. Then $x_{i} x_{j}$ is the indicator that 
$i$ and $j$ are co-members of this group, and this fact may have some effect on their relationship $y_{i, j}$. A positive association between $x_{i} x_{j}$ and $y_{i, j}$ is referred to as homophily, and a negative association as anti-homophily. Quantifying homophily on an observed attribute can be done with a SRRM by creating a dyadic regressor $x_{d, i, j}$ from a nodal regressor $x_{i}$ through multiplication $\left(x_{d, i, j}=x_{i} x_{j}\right)$ or some other operation. However, the possibility that not all relevant nodal attributes are included in a network dataset motivates inclusion of the multiplicative term $\mathbf{u}_{i}^{\top} \mathbf{v}_{j}$, where $\mathbf{u}_{i}$ and $\mathbf{v}_{i}$ represent unobserved latent factors of node $i$ as a sender and receiver of relations, respectively.

These latent factors may be estimated and examined to highlight additional structure in the data beyond that explained by the SRRM. For example, estimates of the $\mathbf{u}_{i}$ 's and $\mathbf{v}_{i}{ }^{\text {'s }}$ of the rank-2 AME fit to the trade data are displayed in Figure 3. Recall that this model includes polity, GDP and geographic distance as regressors, in addition to the additive effects and multiplicative latent factors. The interpretation of the multiplicative factors is that if $\mathbf{u}_{i}$ and $\mathbf{v}_{j}$ are large and in the

same direction, then nodes $i$ and $j$ tend to have observed trade flows larger than $\boldsymbol{\beta}^{\top} \mathbf{x}_{i, j}+a_{i}+b_{j}$, that is, larger than what is predicted by the additive effects alone. As can be seen from the figure, the estimates of the latent factors from these data highlight some geographically related clustering of nodes, in particular, a cluster of Pacific rim countries and a cluster of mostly European countries. These are patterns that, while related to geographic distance, are not well-represented by a single linear relationship between log-trade and log-distance in the regression model.

\section{Transformation models for non-Gaussian networks}

On their original scale, many dyadic variables are not well-represented by a model with Gaussian errors. In some cases, such as with the trade data, a dyadic variable can be transformed so that the Gaussian AME model is reasonable. In other cases, such as with binary, ordinal, discrete or sparse variables, no such transformation is available. Examples of such data include measures of friendship that are binary (not friends/friends) or ordinal (dislike/neutral/like), discrete counts of conflictual events between countries, or the amount of time two people spend on the phone with each other. In this section we describe extensions of the Gaussian AME model to accommodate ordinal dyadic data, where in what follows, ordinal means any outcome for which the possible values can be put in some meaningful order. This includes discrete outcomes (such as binary indicators or counts), ordered qualitative outcomes (such as low/medium/high), and even continuous outcomes. 
The extensions are based on latent variable representations of probit and ordinal probit regression models.

\subsection{Binary and ordinal network data}

Let $\mathbf{S}$ be the observed sociomatrix for a dyadic variable $s_{i, j}$. The simplest type of ordinal dyadic variable is a binary variable indicating the presence of some type of relationship between $i$ and $j$, so that $s_{i, j}=0$ or 1 depending on whether a social link is absent or present, respectively. One approach to quantifying the association between such a binary variable and other variables is with probit regression, which models the probability of a link between $i$ and $j$ as $\Phi\left(\boldsymbol{\beta}^{\top} \mathbf{x}_{i, j}\right)$, where $\Phi$ is the standard normal CDF. As is well known, the probit regression model has a latent variable representation in which $s_{i, j}$ is the binary indicator that some latent normal random variable $y_{i, j} \sim N\left(\boldsymbol{\beta}^{\top} \mathbf{x}_{i, j}, 1\right)$ is greater than zero Albert and Chib, 1993). An ordinary probit regression model corresponds to the $y_{i, j}$ 's being independent, which is generally an inappropriate assumption for network data. However, a model for binary data that does capture the types of network dependencies discussed in the previous section, such as row and column covariance, dyadic correlation, and triadic dependence, can be represented via an AME model for the latent $y_{i, j}$ 's:

$$
\begin{aligned}
& y_{i, j}=\boldsymbol{\beta}^{\top} \mathbf{x}_{i, j}+\mathbf{u}_{i}^{\top} \mathbf{v}_{j}+a_{i}+b_{j}+\epsilon_{i, j} \\
& s_{i, j}=g\left(y_{i, j}\right),
\end{aligned}
$$

where the $a_{i}$ 's $b_{i}$ 's and $\epsilon_{i, j}$ 's follow the SRM covariance model and $g(y)$ is the binary indicator that $y>0$. Absent the multiplicative term $\mathbf{u}_{i}^{\top} \mathbf{v}_{j}$, this is basically a generalized linear mixed effects model. Including the multiplicative term but absent the SRM covariance structure, this model is a type of generalized bilinear regression (Gabriel, 1998). Including both the multiplicative term and the SRM covariance structure yields a regression model for binary social network data that can accommodate second- and third-order dependence patterns.

This probit AME model for binary data extends in a natural way to accommodate ordinal data with more than two levels. As with binary data, we model the observed sociomatrix $\mathbf{S}$ as being a function of a latent sociomatrix $\mathbf{Y}$ that follows a Gaussian AME distribution. Specifically, the model is the same as in Equation 4.1 but with $g$ being a non-decreasing function. Such a model may be viewed as a type of Gaussian transformation model (Bickel and Ritov, 1997). 
One approach to estimation for these models is as follows: For both the probit and ordinal probit models, observation of $\mathbf{S}$ tells us that $\mathbf{Y}$ lies in a certain set, say $\mathbf{Y} \in C(\mathbf{S})$. For the binary probit model, this set is simply given by $C(\mathbf{S})=\left\{\mathbf{Y} \in \mathbb{R}^{n \times n}: \operatorname{sign}\left(y_{i, j}\right)=\operatorname{sign}\left(2 s_{i, j}-1\right)\right\}$, that is, $s_{i, j}=1$

implies $y_{i, j}>0$ and $s_{i, j}=0$ implies $y_{i, j}<0$. For the ordinal probit model, since $g$ is non-decreasing we have $C(\mathbf{S})=\left\{\mathbf{Y} \in \mathbb{R}^{n \times n}: \max _{i^{\prime} j^{\prime}}\left\{y_{i^{\prime}, j^{\prime}}: s_{i^{\prime}, j^{\prime}}<s_{i, j}\right\}<y_{i, j}<\min _{i^{\prime} j^{\prime}}\left\{y_{i^{\prime}, j^{\prime}}: s_{i, j}<s_{i^{\prime} j^{\prime}}\right\}\right\}$. A likelihood based on the knowledge that $\mathbf{Y} \in C(\mathbf{S})$ is given by $L(\boldsymbol{\theta})=\operatorname{Pr}(\mathbf{Y} \in C(\mathbf{S}) \mid \boldsymbol{\theta})$ where $\boldsymbol{\theta}$ are the parameters in the Gaussian AME model for $\mathbf{Y}$. While a closed form expression for this likelihood is unavailable, a Bayesian approach to estimation and inference is feasible via Gibbs sampling by iteratively simulating $\boldsymbol{\theta}$ from its full conditional distribution given $\mathbf{Y}$, then simulating $\mathbf{Y}$ from its conditional distribution given $\boldsymbol{\theta}$ but constrained to lie in $C(\mathbf{S})$. More details are presented in Section 6 .

\subsection{Censored and ranked nomination data}

Data on human social networks are often obtained by asking participants in a study to name and rank a fixed number of people with whom they are friends. Such a survey method is called a fixed ranked nomination (FRN) scheme, and is used in studies of institutions such as schools or businesses. For example, the National Longitudinal Study of Adolescent Health (AddHealth, Harris et al. (2009) ) asked middle and high-school students to nominate and rank up to five members of the same sex as friends, and five members of the opposite sex as friends.

Data obtained from FRN schemes are similar to ordinal data, in that the ranks of a person's friends may be viewed as an ordinal response. However, FRN data are also censored in a complicated way. Consider a study where people were asked to name and rank up to and including their top five friends. If person $i$ nominates five people but doesn't nominate person $j$, then $s_{i, j}$ is censored: The data cannot tell us whether $j$ is $i$ 's sixth best friend, or whether $j$ is not liked by $i$ at all. On the other hand, if person $i$ nominates four people as friends but could have nominated five, then person $i$ 's data are not censored - the absence of a nomination by $i$ of $j$ indicates that $i$ does not consider $j$ a friend.

A likelihood-based approach to modeling FRN data using an AME model was developed in Hoff et al. (2013). Similar to the approach for ordinal dyadic data described above, this methodology treats the observed ranked outcomes $\mathbf{S}$ as a function of an underlying continuous sociomatrix $\mathbf{Y}$ of 
affinities that is generated from an AME model. Letting $m$ be the maximum number of nominations allowed, and coding $s_{i, j} \in\{m, m-1, \ldots, 1,0\}$ so that $s_{i, j}=m$ indicates that $j$ is $i$ 's most liked friend, the FRN likelihood is derived from the following constraints that the observed ranks $\mathbf{S}$ tell us about the underlying dyadic variables $\mathbf{Y}$ :

$$
\begin{aligned}
s_{i, j}>0 & \Rightarrow y_{i, j}>0 \\
s_{i, j}>s_{i, k} & \Rightarrow y_{i, j}>y_{i, k} \\
s_{i, j}=0 \text { and } d_{i}<m & \Rightarrow y_{i, j} \leq 0 .
\end{aligned}
$$

Constraint (4.2) indicates that if $i \operatorname{ranks} j$, then $i$ has a positive relation with $j\left(y_{i, j}>0\right)$, and constraint 4.3 indicates that a higher rank corresponds to a more positive relation. Letting $d_{i} \in\{0, \ldots, m\}$ be the number of people that $i$ ranks, constraint 4.4 indicates that if $i$ could have made additional friendship nominations but chose not to nominate $j$, they then do not consider $j$ a friend. However, if $s_{i, j}=0$ but $d_{i}=m$ then person $i$ 's unranked relationships are censored, and so $y_{i, j}$ could be positive even though $s_{i, j}=0$. In this case, all that is known about $y_{i, j}$ is that it is less than $y_{i, k}$ for any person $k$ ranked by $i$. In summary, observation of $\mathbf{S}$ tells us that $\mathbf{Y} \in C(\mathbf{S})$ where $C(\mathbf{S})$ is defined by conditions $4.2-4.4$. As with the probit and ordinal AME models, Bayesian inference for this transformation model can proceed by iteratively simulating values of the model parameters and the unknown values of $\mathbf{Y}$ from their full conditional distributions.

\section{Comparisons to other models}

Two popular categories of statistical network models are exponentially parameterized random graph models (ERGMs) and latent variables models. Roughly speaking, ERGMs focus on characterizing global, macro-level patterns in a network, while latent variable models describe local, micro-level patterns of relationships among specific nodes. The AME class of model can characterize both global and local patterns, the former via the global parameters $\left\{\boldsymbol{\beta}, \Sigma, \Psi, \sigma^{2}, \rho\right\}$ and the latter via the node-specific factors $\left\{a_{i}, b_{i}, \mathbf{u}_{i}, \mathbf{v}_{i}: i=1, \ldots, n\right\}$.

\subsection{Comparisons to ERGMs}

An ERGM is a probability model for a binary sociomatrix that includes densities of the form $p(\mathbf{Y})=c(\boldsymbol{\theta}) \exp (\boldsymbol{\theta} \cdot \mathbf{t}(\mathbf{Y}))$, where $\mathbf{t}(\mathbf{Y})$ is a vector of sufficient statistics and $\boldsymbol{\theta}$ is a parameter to 
be estimated. Typical applications use a small number of sufficient statistics, often much smaller than the number of nodes, and in this sense the models describe "global" patterns in the data. An exception to this is the not-infrequent inclusion of out- and in-degree statistics that can characterize the differential sociability and popularity of the nodes. For example, one of the first ERGMs to be widely used and studied was the " $p_{1}$ " model (Holland and Leinhardt, 1981) with density

$$
p(\mathbf{Y}) \propto \exp \left(\mu \sum_{i, j} y_{i, j}+\sum_{i}\left(a_{i} \sum_{j} y_{i, j}+b_{i} \sum_{j} y_{j, i}\right)+\rho \sum_{i, j} y_{i, j} y_{j, i}\right),
$$

which includes as sufficient statistics the total number of ties $\sum_{i, j} y_{i, j}$, the number of reciprocated ties $\sum_{i, j} y_{i, j} y_{j, i}$ and the in- and out-degrees $\left\{\sum_{j} y_{i, j}, \sum_{j} y_{j, i}, i=1, \ldots, n\right\}$. The parameters in this model represent roughly the same data features as they do in the SRM: an overall mean of the relations $(\mu)$, heterogeneity in row and column means (the $a_{i}$ 's and $b_{i}$ 's) and dyadic correlation $(\rho)$. Similarities are also found between the SRRM and the " $p_{2}$ " model developed by van Duijn et al. (2004). The $p_{2}$ model extends the $p_{1}$ model by including regressors (as does the SRRM), and additionally treats the node-level parameters $a_{i}$ and $b_{i}$ as potentially correlated random effects (as do the SRM and SRRM).

Holland and Leinhardt (1981) concede that the $p_{1}$ model is of limited utility due to its inability to describe more complex forms of dependency such as transitivity or clustering. While inclusion of appropriate regressors, either in a $p_{2}$ model or SRRM, can represent some degree of higherorder dependency, often such models still exhibit lack-of-fit and more complex models are desired. As described in Section 3, the AME approach is to include a multiplicative latent variable term $\mathbf{u}_{i}^{\top} \mathbf{v}_{j}$, that when thought of as a random effect, induces non-zero third order moments in the error structure. In contrast, the ERGM approach to describing higher-order dependencies is to include additional sufficient statistics, such as the number of triangles observed in the graph, or the number of cycles of various lengths (Snijders et al., 2006). Unfortunately, simultaneous inclusion of such statistics and those that naturally represent degree heterogeneity can lead to model degeneracy (Handcock, 2003, Hunter and Handcock, 2006).

\subsection{Comparison to other latent variable models}

While globally inducing third-order dependence among network outcomes, the multiplicative term $\mathbf{u}_{i}^{\top} \mathbf{v}_{j}$ in the AME model can also be interpreted locally at the micro-level, in that $\mathbf{u}_{i}$ and $\mathbf{v}_{i}$ describe 

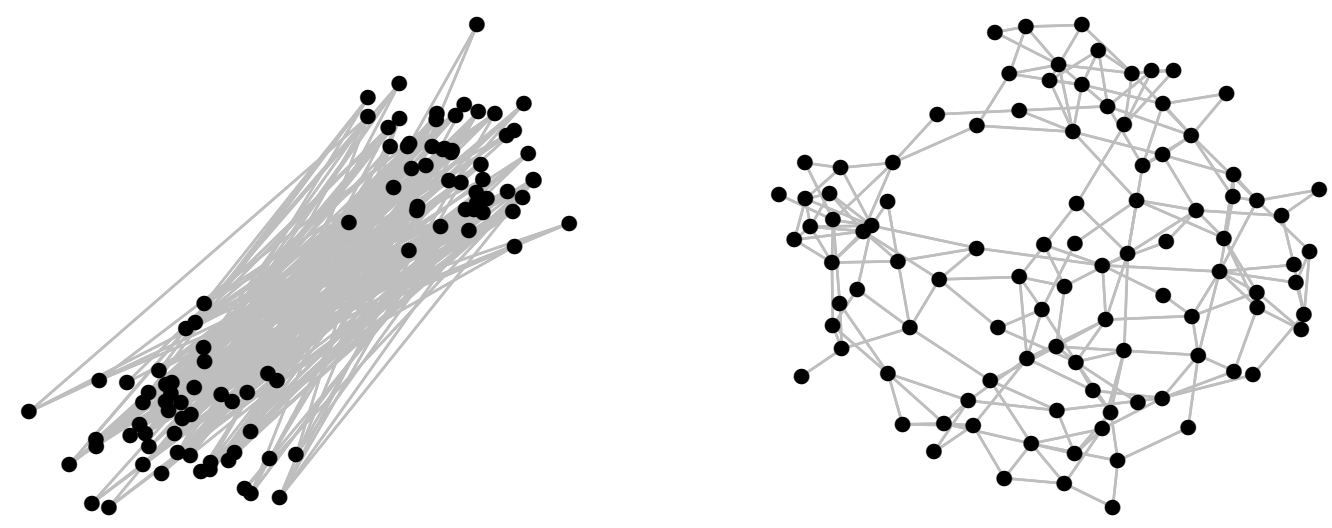

Figure 4: Two hypothetical networks. The network on the left can be represented by two groups of stochastically equivalent nodes. The network on the right can be represented by an embedding of the nodes in two-dimensional Euclidean space.

latent features of node $i$ as a sender and receiver of ties. Estimates of the features (such as those displayed in Figure 3 can be used to identify interesting nodes, assist with visualization of network patterns, or be used as an input to other data analysis methods, such as clustering (Rohe et al. 2011). Other popular non-additive latent variable models for network data include the stochastic blockmodel (Nowicki and Snijders, 2001) and the latent distance model (Hoff et al., 2002). The blockmodel assumes each node belongs to an unobserved latent class or "block", and that the relations between two nodes are determined (statistically) by their block memberships. This model is based on the assumption of stochastic equivalence, that is, the assumption that the nodes can be divided into groups such that members of the same group have the same distribution of relationships to other nodes. In contrast, the distance model assumes each node has some unobserved location in a latent "social space," and that the strength of a relation between two nodes is decreasing in the distance between them in this space. This model provides a compact representation of certain patterns seen in social networks such as transitivity and community, that is, the existence subgroups of nodes with strong within-group relations.

Figure 4 displays two hypothetical symmetric networks, each one of which can be well-represented by one of these two latent variable models. The network on the left can be well-represented by 
a two-group stochastic blockmodel in which the within-group density of ties is lower than the between-group density. Such a network is not representable by a latent distance model because in such a model, stochastic equivalence of two nodes is confounded with the expected strength of their relationship: In a latent distance model, two nodes are stochastically equivalent if they are in the same location in the social space. However, if they are in the same location, then the distance between them is zero and so their expected relationship is strong. As such, networks where stochastically equivalent nodes have weak ties will not be well-represented by a latent distance model. Conversely, the network displayed on the right side of Figure 4 is very well represented by a two-dimensional latent distance model in which the probability of a tie between two nodes is decreasing in the distance between them. However, representation of this network by a blockmodel would require a large number of blocks (e.g. one block in each subregion of the space), none of which would be particularly cohesive or distinguishable from the others.

In contrast to these two extreme networks, real networks exhibit combinations of stochastic equivalence and transitivity in varying amounts. Inference based on either a blockmodel or a distance model would then provide only an incomplete description of the heterogeneity across nodes in terms of how they form ties to others. Fortunately, as shown in Hoff $(2008)$, latent variable models based on multiplicative effects (such as AME models) can represent both of these types of network patterns, and therefore provide a generalization of both the stochastic blockmodel and the latent distance model. To explain this generalization, we consider the simple case of an undirected dyadic variable so that the sociomatrix is symmetric. Each of the three types of latent variable models may be written abstractly as $y_{i, j} \sim m_{i, j}+\alpha\left(\mathbf{u}_{i}, \mathbf{u}_{j}\right)$ where $\alpha$ is some function of the node-specific latent variables $\mathbf{u}_{1}, \ldots, \mathbf{u}_{n}, m_{i, j}$ consists of any other terms in the model (such as a regression term or additive effects), and " $y \sim x$ " means that the distribution of $y$ is stochastically increasing in $x$. The three latent variable models correspond to the following three specifications of the function $\alpha$ :

Stochastic blockmodel: $\alpha\left(\mathbf{u}_{i}, \mathbf{u}_{j}\right)=\mathbf{u}_{i}^{\top} \Theta \mathbf{u}_{j}$, where $\mathbf{u}_{i} \in \mathbb{R}^{r}$ is a standard basis vector indicating block membership, and $\Theta$ is $r \times r$ symmetric.

Latent distance model: $\alpha\left(\mathbf{u}_{i}, \mathbf{u}_{j}\right)=-\left|\mathbf{u}_{i}-\mathbf{u}_{j}\right|$, where $\mathbf{u}_{i} \in \mathbb{R}^{r}$.

Multiplicative effects model: $\alpha\left(\mathbf{u}_{i}, \mathbf{u}_{j}\right)=\mathbf{u}_{i}^{\top} \Lambda \mathbf{u}_{j}$, where $\mathbf{u}_{i} \in \mathbb{R}^{r}$ and $\Lambda$ is an $r \times r$ diagonal matrix. 
Hoff (2008) referred to the symmetric multiplicative effects model as an "eigenmodel", as the matrix $\mathbf{U} \Lambda \mathbf{U}^{\top}$ resembles an eigendecomposition of a rank- $r$ matrix. Note that as the $\mathbf{u}_{i}$ 's range over $r$-dimensional Euclidean space, and $\Lambda$ ranges over all $r \times r$ diagonal matrices, the matrix $\mathbf{U} \Lambda \mathbf{U}^{\top}$ ranges over the space of all symmetric rank- $r$ matrices. Similarly, for the asymmetric AME models discussed elsewhere in this article, as the $\mathbf{u}_{i}$ 's and $\mathbf{v}_{i}$ 's range over $r$-dimensional space, the multiplicative term $\mathbf{U V}^{\top}$ ranges over the space of all $n \times n$ rank- $r$ matrices.

To compare these models we compare the sets of matrices that are representable by their latent variables. Let $\mathcal{S}_{n}$ be the set of $n \times n$ symmetric matrices, and let

$$
\begin{aligned}
& \mathcal{B}_{r}=\left\{\mathbf{S} \in \mathcal{S}_{n}: s_{i, j}=\mathbf{u}_{i}^{\top} \Theta \mathbf{u}_{j}, \mathbf{u}_{i} \text { a standard basis vector }, \Theta \in \mathbb{R}^{r \times r} \text { symmetric }\right\} ; \\
& \mathcal{D}_{r}=\left\{\mathbf{S} \in \mathcal{S}_{n}: s_{i, j}=-\left|\mathbf{u}_{i}-\mathbf{u}_{j}\right|, \mathbf{u}_{i} \in \mathbb{R}^{r}\right\} ; \\
& \mathcal{E}_{r}=\left\{\mathbf{S} \in \mathcal{S}_{n}: s_{i, j}=\mathbf{u}_{i}^{T} \Lambda \mathbf{u}_{j}, \mathbf{u}_{i} \in \mathbb{R}^{r}, \Lambda \text { a } r \times r \text { diagonal matrix }\right\} .
\end{aligned}
$$

In other words, $\mathcal{B}_{r}$ is the set of matrices expressible as a $r$-dimensional blockmodel, and $\mathcal{D}_{r}$ and $\mathcal{E}_{r}$ are defined similarly. Hoff (2008) showed the following:

1. $\mathcal{E}_{r}$ generalizes $\mathcal{B}_{r}$;

2. $\mathcal{E}_{r+1}$ weakly generalizes $\mathcal{D}_{r}$;

3. $\mathcal{D}_{r}$ does not weakly generalize $\mathcal{E}_{1}$.

Result 1 means that $\mathcal{B}_{r}$ is a proper subset of $\mathcal{E}_{r}$ unless $r \geq n$. This is because the matrix $\mathbf{S}$ corresponding to an $r$-group blockmodel is of rank $r$ or less, and $\mathcal{E}_{r}$ includes all such matrices. Result 2 means that for any $\mathbf{S} \in \mathcal{D}_{r}$, there exists an $\tilde{\mathbf{S}} \in \mathcal{E}_{r+1}$ whose elements are a monotonic transformation of those of $\mathbf{S}$, that is, have a numerical order that matches that of the elements of $\mathbf{S}$. Finally, result 3 says that there exist rank-1 matrices $\mathbf{S}$, expressible via one-dimensional multiplicative effects, that cannot be order-matched by a distance model of any dimension. Taken together, these results imply that multiplicative effects models can represent both the types of network patterns representable by stochastic blockmodels and those representable by latent distance models, and so is a more general and flexible class of models than either of these two other latent variable models. See Hoff $(2008)$ for more details and numerical examples. 


\section{Inference via posterior approximation}

While maximum likelihood estimation for a Gaussian AME model is feasible, it is quite challenging for binary, ordinal and other AME transformation models because the likelihoods involve intractable integrals arising from the combination of the transformation and dependencies induced by the SRM. However, reasonably standard Gibbs sampling algorithms can be constructed to provide Bayesian inference for a wide variety of AME network models. We first construct a Gibbs sampler for Gaussian SRRMs, then extend the sampler to accommodate Gaussian AME models, and finally extend the algorithm to fit AME transformation models. These algorithms are implemented in the $\mathrm{R}$ package amen (Hoff et al., 2012). Hoff (2015) provides an $\mathrm{R}$ vignette with several data analysis examples using these methods.

\subsection{Gibbs sampling for the SRRM}

The unknown quantities in the Gaussian SRRM include the parameters $\boldsymbol{\beta}, \Sigma, \sigma^{2}$, and $\rho$, and the random effects $\mathbf{a}$ and $\mathbf{b}$. Posterior approximation for these quantities is facilitated by using a $N_{p}\left(\boldsymbol{\beta}_{0}, \mathbf{Q}_{0}^{-1}\right)$ prior distribution for $\boldsymbol{\beta}$, a gamma $\left(\nu_{0} / 2, \nu_{0} \sigma_{0}^{2} / 2\right)$ prior distribution for $1 / \sigma^{2}$ and a

Wishart $\left(\Sigma_{0}^{-1} / \eta_{0}, \eta_{0}\right)$ prior distribution for $\Sigma^{-1}$. A Gibbs sampler proceeds by iteratively simulating the values of the unknown quantities from their conditional distributions, thereby generating a Markov chain having a stationary distribution equal to the target posterior distribution. Values simulated from this Markov chain can be used to approximate a variety of posterior quantities of interest. Given starting values of the unknown quantities, the algorithm proceeds by iterating the following steps:

1. Simulate $\{\boldsymbol{\beta}, \mathbf{a}, \mathbf{b}\}$ given $\mathbf{Y}, \Sigma, \sigma^{2}, \rho$;

2. Simulate $\sigma^{2}$ given $\mathbf{Y}, \boldsymbol{\beta}, \mathbf{a}, \mathbf{b}, \rho$;

3. Simulate $\rho$ given $\mathbf{Y}, \boldsymbol{\beta}, \mathbf{a}, \mathbf{b}, \sigma^{2}$;

4. Simulate $\Sigma$ given $\mathbf{a}, \mathbf{b}$;

5. Simulate missing values of $\mathbf{Y}$ given $\boldsymbol{\beta}, \mathbf{a}, \mathbf{b}, \sigma^{2}, \rho$ and observed values of $\mathbf{Y}$.

We include the last step because, while sociomatrices typically have undefined diagonals, the calculations below make use of matrix operations that are only defined on matrices with no missing 
values. By treating the diagonal values as missing at random, the fact that they are undefined will not affect the posterior distribution. Additionally, this step permits imputation of other dyadic outcomes that are missing at random.

Steps 2 through 5 are relatively standard. We discuss implementation of these steps before deriving the full conditional distribution of $\{\boldsymbol{\beta}, \mathbf{a}, \mathbf{b}\}$. To implement steps 2 and 3 , consider the stochastic representation of the SRRM as

$$
\mathbf{Y}=\mathbf{M}(\mathbf{X}, \boldsymbol{\beta})+\mathbf{a} \mathbf{1}^{\top}+\mathbf{1} \mathbf{b}^{\top}+\mathbf{E}
$$

where $\mathbf{E}=c \mathbf{Z}+d \mathbf{Z}^{\top}$, with $\mathbf{Z} \sim N_{n \times n}(\mathbf{0}, \mathbf{I}), c=\sigma\left\{(1+\rho)^{1 / 2}+(1-\rho)^{1 / 2}\right\} / 2$ and $d=\sigma\{(1+$ $\left.\rho)^{1 / 2}-(1-\rho)^{1 / 2}\right\} / 2$. Then $\mathbf{E}$ is a mean-zero Gaussian matrix with $\operatorname{Var}\left[\left(\begin{array}{c}e_{i, j} \\ e_{j, i}\end{array}\right)\right]=\sigma^{2}\left(\begin{array}{ll}1 & \rho \\ \rho & 1\end{array}\right) \equiv \Sigma_{e}$ and $\operatorname{Var}\left[e_{i, i}\right]=\sigma^{2}(1+\rho)$, with the elements of $\mathbf{E}$ being otherwise independent. Now given $\boldsymbol{\beta}$, a and $\mathbf{b}$, construct $\mathbf{E}=\mathbf{Y}-\left(\mathbf{M}(\mathbf{X}, \boldsymbol{\beta})+\mathbf{a} \mathbf{1}^{\top}+\mathbf{1} \mathbf{b}^{\top}\right)$. As a function of $\sigma^{2}$ and $\rho$, the density of $\mathbf{E}$ is proportional to

$$
\left(\sigma^{2}\right)^{-n^{2} / 2}\left(1-\rho^{2}\right)^{-\left(\begin{array}{c}
n \\
2
\end{array}\right) / 2}(1+\rho)^{-n / 2} \times \exp \left\{-\left(S S_{1}+S S_{2}\right) /\left[2 \sigma^{2}\right]\right\}
$$

where $\left.S S_{1}=\sum_{i<j}\left(\begin{array}{c}e_{i, j} \\ e_{j, i}\end{array}\right)^{\top}\left(\begin{array}{cc}1 & \rho \\ \rho & 1\end{array}\right)^{-1}\left(\begin{array}{c}e_{i, j} \\ e_{j, i}\end{array}\right)\right\}$ and $S S_{2}=\sum_{i=1}^{n} e_{i, i}^{2} /(1+\rho)$. The full conditional distribution of $1 / \sigma^{2}$ is therefore gamma $\left(\left[\nu_{0}+n^{2}\right] / 2,\left[\nu_{0} \sigma_{0}^{2}+S S_{1}+S S_{2}\right] / 2\right)$. As for $\rho$, we do not know of a standard semiconjugate prior distribution. However, $\rho$ is just a scalar parameter bounded between -1 and +1 , and so approximate simulation of $\rho$ from its full conditional distribution (given an arbitrary prior distribution) could be achieved by computing the unnormalized posterior density on a grid of values, or by slice sampling, or instead using a Metropolis-Hastings updating procedure.

To update $\Sigma$ in step 4 , let $\mathbf{f}_{i}=\left(\mathbf{a}_{i}, \mathbf{b}_{i}\right)$ and recall that the random effects model for the $\mathbf{f}_{i}$ 's is that $\mathbf{f}_{1}, \ldots, \mathbf{f}_{n} \sim$ i.i.d. $N_{2}(0, \Sigma)$. Given a Wishart prior distribution for $\Sigma^{-1}$, the conditional distribution of $\Sigma^{-1}$ given $\mathbf{f}_{1}, \ldots, \mathbf{f}_{n}$ is $\operatorname{Wishart}\left(\left[\eta_{0} \Sigma_{0}+\mathbf{F}^{\top} \mathbf{F}\right]^{-1}, \eta_{0}+n\right)$, where $\mathbf{F}$ is the $n \times 2$ matrix with $i$ th row equal to $\mathbf{f}_{i}$.

The missing entries of $\mathbf{Y}$ may be updated by simulating from their full conditional distributions. The full conditional distribution of diagonal entry $y_{i, i}$ is $N\left(m_{i, j}+a_{i}+b_{j}, \sigma^{2}(1+\rho)\right)$. If a dyadic pair of outcomes $\left(y_{i, j}, y_{j, i}\right)$ is missing, then its full conditional distribution is bivariate normal with mean vector $\left(m_{i, j}+a_{i}+b_{j}, m_{j, i}+a_{j}+b_{i}\right)$ and covariance matrix $\left.\sigma^{2}\left(\begin{array}{ll}1 & \rho \\ \rho & 1\end{array}\right)\right)$. However, if $y_{i, j}$ is observed and $y_{j, i}$ is not, then the full conditional distribution of $y_{j, i}$ is normal with mean $\rho \times\left(y_{i, j}-m_{i, j}-a_{i}-b_{j}\right)+m_{j, i}+a_{j}+b_{i}$ and variance $\sigma^{2}\left(1-\rho^{2}\right)$. 
Step 1 of the Gibbs sampler requires simulation of $\{\boldsymbol{\beta}, \mathbf{a}, \mathbf{b}\}$ from its joint distribution given $\mathbf{Y}, \Sigma, \sigma^{2}$, and $\rho$. This is challenging because of the dyadic correlation. However, calculations are simplified by transforming $\mathbf{Y}$ so that the dyadic correlation is zero: Given values of $\sigma^{2}$ and $\rho$, we may construct $\tilde{\mathbf{Y}}=\tilde{c} \mathbf{Y}+\tilde{d} \mathbf{Y}^{\top}$, where $\tilde{c}=\left\{(1+\rho)^{-1 / 2}+(1-\rho)^{-1 / 2}\right\} /(2 \sigma)$ and $\tilde{d}=$ $\left\{(1+\rho)^{-1 / 2}-(1-\rho)^{-1 / 2}\right\} /(2 \sigma)$. It follows that

$$
\tilde{\mathbf{Y}} \stackrel{d}{=} \mathbf{M}(\tilde{\mathbf{X}}, \boldsymbol{\beta})+\tilde{\mathbf{a}} \mathbf{1}^{\top}+\mathbf{1} \tilde{\mathbf{b}}^{\top}+\mathbf{Z}
$$

where $\mathbf{Z} \sim N_{n \times n}(\mathbf{0}, \mathbf{I}), \tilde{\mathbf{x}}_{i, j}=\tilde{c} \mathbf{x}_{i, j}+\tilde{d} \mathbf{x}_{j, i},\left(\tilde{a}_{1}, \tilde{b}_{1}\right), \ldots,\left(\tilde{a}_{n}, \tilde{b}_{n}\right) \sim$ i.i.d $N_{2}(\mathbf{0}, \tilde{\Sigma})$ with $\tilde{\Sigma}=$ $\Sigma_{e}^{-1 / 2} \Sigma \Sigma_{e}^{-1 / 2}$. Therefore, simulation of $\{\boldsymbol{\beta}, \mathbf{a}, \mathbf{b}\}$ from its conditional distribution given $\mathbf{Y}, \Sigma, \sigma^{2}$ and $\rho$ may be accomplished as follows:

1.a Compute $\tilde{\mathbf{Y}}, \tilde{\mathbf{X}}$ and $\tilde{\Sigma}=\Sigma_{e}^{-1 / 2} \Sigma \Sigma_{e}^{-1 / 2}$

1.b Simulate $\{\boldsymbol{\beta}, \tilde{\mathbf{a}}, \tilde{\mathbf{b}}\}$ from its conditional distribution based on 6.2 ;

1.c Set $\left(\begin{array}{c}a_{i} \\ b_{i}\end{array}\right)=\Sigma_{e}^{1 / 2}\left(\begin{array}{c}\tilde{a}_{i} \\ \tilde{b}_{i}\end{array}\right)$ for $i=1, \ldots, n$.

Step 1.b may be implemented by simulating $\boldsymbol{\beta}$ conditional on $\{\tilde{\mathbf{Y}}, \tilde{\mathbf{X}}, \tilde{\Sigma}\}$ and then simulating $\{\tilde{\mathbf{a}}, \tilde{\mathbf{b}}\}$ conditional on $\boldsymbol{\beta}$ and $\{\tilde{\mathbf{Y}}, \tilde{\mathbf{X}}, \tilde{\Sigma}\}$. We first derive the latter distribution, as it facilitates the derivation of the former. For notational simplicity, we drop the tildes on the symbols.

Let $\mathbf{Y}=\mathbf{M}+\mathbf{a} \mathbf{1}^{\top}+\mathbf{1} \mathbf{b}^{\top}+\mathbf{Z}$ where the elements of $\mathbf{Z}$ are i.i.d. standard normal random variables, and let $\mathbf{f}=(\mathbf{a}, \mathbf{b})$ be the concatenation of $\mathbf{a}$ and $\mathbf{b}$ so that $\mathbf{f} \sim N_{2 n}(\mathbf{0}, \Sigma \otimes \mathbf{I})$, where " $\otimes$ " denotes the Kronecker product. Vectorizing the formula for $\mathbf{Y}$ gives $\mathbf{y}=\mathbf{m}+[(\mathbf{1} \otimes \mathbf{I})(\mathbf{I} \otimes \mathbf{1})] \mathbf{f}+\mathbf{z}$. Let $\mathbf{r}=\mathbf{y}-\mathbf{m}$ and $\mathbf{W}=[(\mathbf{1} \otimes \mathbf{I})(\mathbf{I} \otimes \mathbf{1})]$. The conditional density of $\mathbf{f}$ given $\mathbf{r}$ and $\Sigma$ is given by

$$
\begin{aligned}
p(\mathbf{f} \mid \mathbf{r}, \Sigma) & \propto \exp \left(-(\mathbf{r}-\mathbf{W} \mathbf{f})^{\top}(\mathbf{r}-\mathbf{W} \mathbf{f}) / 2\right) \times \exp \left(-\mathbf{f}^{\top}\left(\Sigma^{-1} \otimes \mathbf{I}\right) \mathbf{f} / 2\right) \\
& \propto \exp \left(-\mathbf{f}^{\top}\left[\mathbf{W}^{\top} \mathbf{W}+\Sigma^{-1} \otimes \mathbf{I}\right] \mathbf{f} / 2+\mathbf{f}^{\top} \mathbf{W}^{\top} \mathbf{r}\right) .
\end{aligned}
$$

This is the kernel of a multivariate normal distribution with variance $\operatorname{Var}[\mathbf{f} \mid \mathbf{r}]=\left(\mathbf{W}^{\top} \mathbf{W}+\Sigma^{-1} \otimes \mathbf{I}\right)^{-1}$ and expectation $\mathrm{E}[\mathbf{f} \mid \mathbf{r}]=\left(\mathbf{W}^{\top} \mathbf{W}+\Sigma^{-1} \otimes \mathbf{I}\right)^{-1} \mathbf{W}^{\top} \mathbf{r}$. Some matrix manipulations yield $\operatorname{Var}[\mathbf{f} \mid \mathbf{r}]=$ $\mathbf{G} \otimes \mathbf{I}-\mathbf{H} \otimes \mathbf{1 1 ^ { \top }}$, where

- $\mathbf{G}=\left(\Sigma^{-1}+n \mathbf{I}\right)^{-1}$

- $\mathbf{H}=\left(\Sigma^{-1}+n \mathbf{1 1} \mathbf{1}^{\top}\right)^{-1}\left(\begin{array}{ll}0 & 1 \\ 1 & 0\end{array}\right) \mathbf{G}$. 
Now let $\mathbf{s}=\mathbf{W}^{\top} \mathbf{r}=\left(\mathbf{1}^{\top} \mathbf{R}^{\top}, \mathbf{1}^{\top} \mathbf{R}\right)$, the concatenation of the row sums and column sums of $\mathbf{R}=\mathbf{Y}-\mathbf{M}$. We then have $\mathrm{E}[\mathbf{f} \mid \mathbf{r}]=(\mathbf{G} \otimes \mathbf{I}) \mathbf{s}-\left(\mathbf{H} \otimes \mathbf{1 1}^{\top}\right) \mathbf{s}$. Writing this in terms of the $n \times 2$ matrix $\mathbf{F}$ whose vectorization is $\mathbf{f}$, we have $\mathrm{E}[\mathbf{F} \mid \mathbf{R}]=\mathbf{S G}-t \mathbf{1 1}{ }^{\top} \mathbf{H}$, where $\mathbf{S}$ is the $n \times 2$ matrix whose first and second columns are the row and column sums of $\mathbf{R}$, respectively, and $t=\mathbf{1}^{\top} \mathbf{R} \mathbf{1}$, the sum total of the entries of $\mathbf{R}$. Therefore, to simulate $\mathbf{F}$ (and hence $\mathbf{a}$ and $\mathbf{b}$ ) from its full conditional distribution, we set $\mathbf{F}$ equal to

$$
\mathbf{F}=\left(\mathbf{S G}-t \mathbf{1 1} \mathbf{1}^{\top} \mathbf{H}\right)+\mathbf{E}
$$

where $\mathbf{E}$ is a simulated $n \times 2$ normal matrix with mean zero and variance $\mathbf{G} \otimes \mathbf{I}-\mathbf{H} \otimes \mathbf{1 1}^{\top}$. To simulate this normal matrix, rewrite $\operatorname{Var}[\mathbf{f} \mid \mathbf{r}]$ as $\operatorname{Var}[\mathbf{f} \mid \mathbf{r}]=[\mathbf{G}-n \mathbf{H}] \otimes \mathbf{I}+n \mathbf{H} \otimes\left[\mathbf{I}-\mathbf{1 1}^{\top} / n\right]$, and recognize this as the covariance matrix of

$$
\mathbf{Z}_{1}(\mathbf{G}-n \mathbf{H})^{1 / 2}+\left(\mathbf{I}-\mathbf{1 1}^{\top} / n\right) \mathbf{Z}_{2}(\sqrt{n} \mathbf{H})^{1 / 2}
$$

where $\mathbf{Z}_{1}$ and $\mathbf{Z}_{2}$ are both $n \times 2$ matrices of standard normal entries. To summarize, to simulate F from its full conditional distribution,

1. Simulate two $n \times 2$ matrices $\mathbf{Z}_{1}$ and $\mathbf{Z}_{2}$ with i.i.d. standard normal entries;

2. Compute $\mathbf{E}=\mathbf{Z}_{1}(\mathbf{G}-n \mathbf{H})^{1 / 2}+\left(\mathbf{I}-\mathbf{1 1}^{\top} / n\right) \mathbf{Z}_{2}(\sqrt{n} \mathbf{H})^{1 / 2}$

3. Set $\mathbf{F}=\left(\mathbf{S G}-t \mathbf{1 1} \mathbf{1}^{\top} \mathbf{H}\right)+\mathbf{E}$.

We can use this result to obtain the conditional distribution of $\boldsymbol{\beta}$ given $\mathbf{y}$ and $\Sigma$ (but unconditional on $\mathbf{a}, \mathbf{b})$. The density of this distribution is proportional to $p(\mathbf{y} \mid \boldsymbol{\beta}, \Sigma) \pi(\boldsymbol{\beta})$, the product of the SRRM likelihood and the prior density for $\boldsymbol{\beta}$. The SRRM likelihood may be obtained using Bayes' rule, $p(\mathbf{y} \mid \boldsymbol{\beta}, \Sigma)=p(\mathbf{y} \mid \boldsymbol{\beta}, \mathbf{a}, \mathbf{b}) p(\mathbf{a}, \mathbf{b} \mid \Sigma) / p(\mathbf{a}, \mathbf{b} \mid \mathbf{y}, \boldsymbol{\beta}, \Sigma)$. The terms on the right side of this equation are easily available: $p(\mathbf{y} \mid \boldsymbol{\beta}, \mathbf{a}, \mathbf{b})$ is the product of univariate normal densities corresponding to $y_{i, j} \sim N\left(\boldsymbol{\beta}^{\top} \mathbf{x}_{i, j}+a_{i}+b_{j}, 1\right)$ independently across ordered pairs. The terms $p(\mathbf{a}, \mathbf{b} \mid \Sigma)$ and $p(\mathbf{a}, \mathbf{b} \mid \mathbf{y}, \boldsymbol{\beta}, \Sigma)$ are the prior and full conditional distributions of $(\mathbf{a}, \mathbf{b})$, the latter having been obtained in the previous paragraph. Putting these terms together and simplifying yields the following form for the uncorrelated SRRM likelihood:

$$
\begin{gathered}
p(\mathbf{y} \mid \boldsymbol{\beta}, \Sigma)=(2 \pi)^{-n^{2} / 2}|\mathbf{I}+n \Sigma|^{-(n-1) / 2}\left|\mathbf{I}+n \Sigma \mathbf{1 1}^{\top}\right|^{-1 / 2} \times \\
\exp \left\{-\left(\mathbf{r}^{\top} \mathbf{r}+t^{2} \mathbf{1}^{\top} \mathbf{H} \mathbf{1}-\operatorname{tr}\left(\mathbf{S}^{\top} \mathbf{S G}\right)\right) / 2\right\} .
\end{gathered}
$$


This is quadratic in the $r_{i, j}$ 's, and hence also quadratic in $\boldsymbol{\beta}$. Some algebra gives

$$
p(\mathbf{y} \mid \boldsymbol{\beta}, \Sigma) \propto \exp \left\{-\boldsymbol{\beta}^{\top}\left(\mathbf{Q}_{1}+\mathbf{Q}_{2}+\mathbf{Q}_{3}\right) \boldsymbol{\beta} / 2+\boldsymbol{\beta}^{\top}\left(\boldsymbol{\ell}_{1}+\boldsymbol{\ell}_{2}+\boldsymbol{\ell}_{3}\right)\right\}
$$

where $\mathbf{Q}_{1}=\mathbf{X}^{\top} \mathbf{X}$ and $\boldsymbol{\ell}_{1}=\mathbf{X}^{\top} \mathbf{y}$, with $\mathbf{X}$ being the $n^{2} \times p$ matrix of the $\mathbf{x}_{i, j}$ 's $; \mathbf{Q}_{2}=n^{4} h \overline{\mathbf{x}} \overline{\mathbf{x}}^{\top}$ and $\boldsymbol{\ell}_{2}=n^{4} h \overline{\mathbf{x}} \bar{y}$ with $h=\mathbf{1}^{\top} \mathbf{H} \mathbf{1}, \overline{\mathbf{x}}$ being the average of the $\mathbf{x}_{i, j}$ 's and $\bar{y}$ being the average of the $y_{i, j}$ 's, and

$$
\begin{aligned}
\mathbf{Q}_{3} & =-n^{2}\left(g_{11} \overline{\mathbf{X}}_{r}^{\top} \overline{\mathbf{X}}_{r}+g_{12}\left(\overline{\mathbf{X}}_{r}^{\top} \overline{\mathbf{X}}_{c}+\overline{\mathbf{X}}_{c}^{\top} \overline{\mathbf{X}}_{r}\right)+g_{22} \overline{\mathbf{X}}_{c}^{\top} \overline{\mathbf{X}}_{c}\right) \\
\boldsymbol{\ell}_{3} & =-n^{2}\left(g_{11} \overline{\mathbf{X}}_{r}^{\top} \overline{\mathbf{y}}_{r}+g_{12}\left(\mathbf{X}_{r}^{\top} \overline{\mathbf{y}}_{c}+\overline{\mathbf{X}}_{c}^{\top} \overline{\mathbf{y}}_{r}\right)+g_{22} \overline{\mathbf{X}}_{c}^{\top} \overline{\mathbf{y}}_{c}\right),
\end{aligned}
$$

where $\overline{\mathbf{y}}_{r}$ is the $n \times 1$ vector of row means of $\mathbf{Y}, \overline{\mathbf{X}}_{r}$ is the $n \times p$ matrix whose $i$ th row is the average of $\mathbf{x}_{i, j}$ over $j=1, \ldots, n$, and $\overline{\mathbf{y}}_{c}$ and $\overline{\mathbf{X}}_{c}$ are analogously defined as column means. Now the prior density for $\boldsymbol{\beta}$ is proportional to $\exp \left\{-\boldsymbol{\beta}^{\top} \mathbf{Q}_{0} \boldsymbol{\beta} / 2+\boldsymbol{\beta}^{\top} \mathbf{Q}_{0} \boldsymbol{\beta}_{0}\right\}$, and so the conditional density is given by

$$
p(\boldsymbol{\beta} \mid \mathbf{y}, \Sigma) \propto p(\mathbf{y} \mid \boldsymbol{\beta}, \Sigma) \times \pi(\boldsymbol{\beta}) \propto \exp \left\{-\boldsymbol{\beta}^{\top}\left(\mathbf{Q}_{0}+\mathbf{Q}\right) \boldsymbol{\beta} / 2+\boldsymbol{\beta}^{\top}\left(\mathbf{Q}_{0} \boldsymbol{\beta}_{0}+\boldsymbol{\ell}\right)\right\}
$$

where $\mathbf{Q}=\mathbf{Q}_{1}+\mathbf{Q}_{2}+\mathbf{Q}_{3}$ and $\boldsymbol{\ell}=\boldsymbol{\ell}_{1}+\boldsymbol{\ell}_{2}+\boldsymbol{\ell}_{3}$. This is a multivariate normal density, with variance $\left(\mathbf{Q}_{0}+\mathbf{Q}\right)^{-1}$ and mean $\left(\mathbf{Q}_{0}+\mathbf{Q}\right)^{-1}\left(\mathbf{Q}_{0} \boldsymbol{\beta}_{0}+\ell\right)$.

\subsection{Gibbs sampling for the AME}

Now suppose that $\mathbf{Y}$ follows a Gaussian AME model, so that $\mathbf{Y}=\mathbf{M}(\mathbf{X}, \boldsymbol{\beta})+\mathbf{U V}^{\top}+\mathbf{a} \mathbf{1}^{\top}+$ $\mathbf{1} \mathbf{b}^{\top}+\mathbf{E}$ where the distribution of $\{\mathbf{a}, \mathbf{b}, \mathbf{E}\}$ follows the social relations covariance model with parameters $\left\{\Sigma, \sigma^{2}, \rho\right\}$. Let $\left(\mathbf{u}_{i}, \mathbf{v}_{i}\right) \sim N_{2 r}(\mathbf{0}, \Psi)$ independently across nodes, and let $\Psi^{-1} \sim$ Wishart $\left(\Psi_{0}^{-1} / \kappa_{0}, \kappa_{0}\right)$ a priori. The joint posterior distribution of the unknown parameters may be approximated by a Gibbs sampler that iterates the following steps:

1. Update $\left(\boldsymbol{\beta}, \mathbf{a}, \mathbf{b}, \sigma^{2}, \rho, \Sigma\right)$ and the missing values of $\mathbf{Y}$ using the algorithm described in Section 6.1 , but with $\mathbf{Y}$ replaced by $\mathbf{Y}-\mathbf{U V}^{\top}$;

2. Simulate $\Psi^{-1} \sim \operatorname{Wishart}\left(\left(\Psi_{0} \kappa_{0}+[\mathbf{U} \mathbf{V}]^{\top}[\mathbf{U} \mathbf{V}]\right)^{-1}, \kappa_{0}+n\right)$, where $[\mathbf{U} \mathbf{V}]$ is the $n \times 2 r$ matrix equal to the column-wise concatenation of $\mathbf{U}$ and $\mathbf{V}$;

3. For each $k=1, \ldots, r$, simulate the $r$ th columns of $\mathbf{U}$ and $\mathbf{V}$ from their full conditional distributions; 
To perform step 3, first consider the full conditional distribution of $\mathbf{u}_{1}$, the first column of $\mathbf{U}$. Let $\mathbf{R}=\mathbf{Y}-\left(\mathbf{M}(\mathbf{X}, \boldsymbol{\beta})+\sum_{k=2}^{r} \mathbf{u}_{k} \mathbf{v}_{k}^{\top}+\mathbf{a} \mathbf{1}^{\top}+\mathbf{1} \mathbf{b}^{\top}\right)$. Then we have $\mathbf{R}=\mathbf{u}_{1} \mathbf{v}_{1}^{\top}+\mathbf{E}$. Decorrelating gives $\tilde{\mathbf{R}}=\tilde{c} \mathbf{R}+\tilde{d} \mathbf{R}=\tilde{c} \mathbf{u}_{1} \mathbf{v}_{1}^{\top}+\tilde{d} \mathbf{v}_{1} \mathbf{u}_{1}^{\top}+\mathbf{Z}$, and vectorizing gives $\tilde{\mathbf{r}}=\left[\tilde{c}\left(\mathbf{v}_{1} \otimes \mathbf{I}\right)+\tilde{d}\left(\mathbf{I} \otimes \mathbf{v}_{1}\right)\right] \mathbf{u}_{1}+\mathbf{z}$. Given $\mathbf{v}_{1}$, this is a linear regression model with outcome vector $\tilde{\mathbf{r}}$, design matrix $\mathbf{W}=\left[\tilde{c}\left(\mathbf{v}_{1} \otimes \mathbf{I}\right)+\tilde{d}\left(\mathbf{I} \otimes \mathbf{v}_{1}\right)\right]$, regression parameters $\mathbf{u}_{1}$, and i.i.d. standard normal errors. Let $\boldsymbol{\mu}_{u \mid v}$ and $\Sigma_{u \mid v}$ be the conditional mean and variance of $\mathbf{u}_{1}$ given $\mathbf{v}_{1}$. Then the conditional distribution of $\mathbf{u}_{1}$ given $\mathbf{v}_{1}$ and $\tilde{\mathbf{R}}$ is normal with mean and variance given by

$$
\begin{aligned}
\operatorname{Var}\left[\mathbf{u}_{1} \mid \tilde{\mathbf{R}}, \mathbf{v}_{1}\right] & =\left(\Sigma_{u \mid v}^{-1}+\mathbf{W}^{\top} \mathbf{W}\right)^{-1} \\
\mathrm{E}\left[\mathbf{u}_{1} \mid \tilde{\mathbf{R}}, \mathbf{v}_{1}\right] & =\left(\Sigma_{u \mid v}^{-1}+\mathbf{W}^{\top} \mathbf{W}\right)^{-1}\left(\Sigma_{u \mid v}^{-1} \boldsymbol{\mu}_{u \mid v}+\mathbf{W}^{\top} \tilde{\mathbf{r}}\right)
\end{aligned}
$$

Some calculations show that $\mathbf{W}^{\top} \mathbf{W}=\left(\tilde{c}^{2}+\tilde{d}^{2}\right)\left\|\mathbf{v}_{1}\right\|^{2} \mathbf{I}+2 \tilde{c} \tilde{d} \mathbf{v}_{1} \mathbf{v}_{1}^{\top}$ and $\mathbf{W}^{\top} \tilde{\mathbf{r}}=\left(\tilde{c} \tilde{\mathbf{R}}+\tilde{d} \tilde{\mathbf{R}}^{\top}\right) \mathbf{v}_{1}$. The full conditional distribution of $\mathbf{v}_{1}$, and the other columns of $\mathbf{U}$ and $\mathbf{V}$, may be obtained similarly.

\subsection{Gibbs sampling for transformation models}

A transformation model assumes that the sociomatrix $\mathbf{S}$ is a function of a latent sociomatrix $\mathbf{Y}$ that follows a Gaussian AME model with parameters $\boldsymbol{\theta}=(\boldsymbol{\beta}, \mathbf{a}, \mathbf{b}, \mathbf{U}, \mathbf{V}, \rho, \Sigma, \Psi)$. This collection of parameters does not include $\sigma^{2}$, because for probit models in general and for the other transformation models described in this article, the overall scale of the $y_{i, j}$ 's is not identifiable, and so we fix $\sigma^{2}=1$. For the transformation models discussed in Section 4, observation of $\mathbf{S}$ implies that $\mathbf{Y} \in C(\mathbf{S})$. Given starting values of $\mathbf{Y}$ and $\boldsymbol{\theta}$, a Gibbs sampler for approximating the joint posterior distribution of $\mathbf{Y}$ and $\boldsymbol{\theta}$ conditional on $\mathbf{S}$ proceeds by iterating the following steps:

1. Update $\boldsymbol{\theta}$ conditional on $\mathbf{Y}$ with the algorithm described in Section 6.2;

2. Update $\mathbf{Y}$ conditional on $\boldsymbol{\theta}$ and $\mathbf{Y} \in C(\mathbf{S})$.

To perform step 2 of this algorithm, first consider the simple probit transformation model where the observed outcome $s_{i, j}$ is the binary indicator that the latent Gaussian variable $y_{i, j}$ is greater than zero. Let $\mu_{i, j}=\boldsymbol{\beta}^{\top} \mathbf{x}_{i, j}+\mathbf{u}_{i}^{\top} \mathbf{v}_{j}+a_{i}+b_{j}$. Then unconditional on $\mathbf{S}$ but given the other parameters, we have that

$$
\left(\begin{array}{l}
y_{i, j} \\
y_{j, i}
\end{array}\right) \sim N_{2}\left(\left(\begin{array}{l}
\mu_{i, j} \\
\mu_{j, i}
\end{array}\right),\left(\begin{array}{ll}
1 & \rho \\
\rho & 1
\end{array}\right)\right)
$$


independently across dyads, and that $y_{i, i} \sim N\left(\mu_{i, i}, 1+\rho\right)$ independently across diagonal entries. Since the diagonal entries of $\mathbf{S}$ are undefined and the diagonal entries of $\mathbf{Y}$ are uncorrelated with the off-diagonal entries, each $y_{i, i}$ value may be updated from its $N\left(\mu_{i, j}, 1+\rho\right)$ distribution. The offdiagonal entries may be updated in two steps: first updating the elements of $\mathbf{Y}$ below the diagonal, and then updating those above. To do so, note that $y_{i, j} \mid y_{j, i} \sim N\left(\mu_{i, j}+\rho \times\left(y_{j, i}-\mu_{j, i}\right), 1-\rho^{2}\right)$. Now in the case of a probit AME model where $s_{i, j}$ is the indicator that $y_{i, j}$ is greater than zero,

the full conditional distribution of $y_{i, j}$ is $N\left(\mu_{i, j}+\rho\left(y_{j, i}-\mu_{j, i}\right), 1-\rho^{2}\right)$ but constrained to be above zero if $y_{i, j}=1$ and below zero otherwise. The full conditional distributions under other types of transformation models are also constrained normal distributions, where the constraint depends on the type of transformation. Univariate constrained normal distributions may be easily simulated from using the inverse-CDF method.

\section{Discussion}

The AME framework is a modular approach for network data analysis based on three statistical models: the social relations covariance model, low-rank matrix representations via multiplicative factors, and Gaussian transformation models. Separately, each of these should be familiar to an applied statistician or data analyst: The first is a type of linear random effects model, the second is analogous to a model-based singular value decomposition, and the third forms the basis of many binary and ordinal regression models. Together, they provide a flexible model-based framework for inference that accounts for many statistical dependencies often found in network data, and accommodates a variety of types of dyadic and nodal variables. Current and future work in this area includes generalizing this framework to analyze datasets from more modern network studies that include multiple sociomatrices on one or more nodesets, such as comparison studies across multiple populations, multiple time points, multiple dyadic variables, or combinations of these. Some steps in this direction have been taken by representing a set of sociomatrices as a tensor (Hoff, 2011, 2016), but these methods are not yet general enough to encompass the wide variety of multivariate, multilevel and longitudinal network datasets that are becoming more prevalent. What is needed is a broad framework like that which is provided for generalized linear mixed models by the nlme or lme4 software (Pinheiro and Bates, 2000; Walker et al., 2015), whereby a data analyst may separately select the type of data being analyzed (continuous, binary, count, 
etc.) and build a complicated model of dependence relationships between subsets of the data. One challenge to developing such a framework for network data is computational - the Gibbs samplers described in this article and implemented in the $\mathrm{R}$ package amen become cumbersome when the number of nodes is above a few thousand, and other integral approximation methods (such as Laplace approximations) for AME transformation models are infeasible because of the complicated dependence induced by the SRM. Fast, stable parameter estimation for large network datasets may require abandoning use of the full likelihood, and instead use composite likelihood estimation (Lindsay, 1988) or modern method-of-moments approaches (Perry, 2017).

\section{Acknowledgments}

This research was partially supported by NSF grant DMS-1505136.

\section{References}

Albert, J. H. and S. Chib (1993). Bayesian analysis of binary and polychotomous response data. J. Amer. Statist. Assoc. 88(422), 669-679.

Baier, S. L. and J. H. Bergstrand (2009). Bonus vetus ols: A simple method for approximating international trade-cost effects using the gravity equation. Journal of International Economics 7r(1), $77-85$.

Bergstrand, J. H. (1985). The gravity equation in international trade: some microeconomic foundations and empirical evidence. The review of economics and statistics 67(3), 474-481.

Bickel, P. J. and Y. Ritov (1997). Local asymptotic normality of ranks and covariates in transformation models. In Festschrift for Lucien Le Cam, pp. 43-54. New York: Springer.

Bond, C. F. and B. R. Lashley (1996). Round-robin analysis of social interaction: Exact and estimated standard errors. Psychometrika 61(2), 303-311.

Bradu, D. and K. R. Gabriel (1974). Simultaneous statistical inference on interactions in two-way analysis of variance. J. Amer. Statist. Assoc. 69, 428-436.

Gabriel, K. R. (1998). Generalised bilinear regression. Biometrika 85(3), 689-700. 
Gill, P. S. and T. B. Swartz (2001). Statistical analyses for round robin interaction data. Canad. J. Statist. 29(2), 321-331.

Gollob, H. F. (1968). A statistical model which combines features of factor analytic and analysis of variance techniques. Psychometrika 33, 73-115.

Handcock, M. S. (2003). Assessing degeneracy in statistical models of social networks. Technical Report 39, Center for Statistics and the Social Sciences, University of Washington.

Harris, K., C. Halpern, E. Whitsel, J. Hussey, J. Tabor, P. Entzel, and J. Udry (2009). The national longitudinal study of adolescent health: Research design.

Hoff, P. (2008). Modeling homophily and stochastic equivalence in symmetric relational data. In J. Platt, D. Koller, Y. Singer, and S. Roweis (Eds.), Advances in Neural Information Processing Systems 20, pp. 657-664. Cambridge, MA: MIT Press.

Hoff, P. (2015). Dyadic data analysis with amen. Technical Report 638, Department of Statistics, University of Washington.

Hoff, P., B. Fosdick, A. Volfovsky, and Y. He (2012). amen: Additive and multiplicative effects modeling of networks and relational data.

Hoff, P., B. Fosdick, A. Volfovsky, and K. Stovel (2013). Likelihoods for fixed rank nomination networks. Network Science 1(3), 253-277.

Hoff, P. D. (2005). Bilinear mixed-effects models for dyadic data. J. Amer. Statist. Assoc. 100(469), $286-295$.

Hoff, P. D. (2009). Multiplicative latent factor models for description and prediction of social networks. Computational and Mathematical Organization Theory 15(4), 261-272.

Hoff, P. D. (2011). Hierarchical multilinear models for multiway data. Computational Statistics $\mathfrak{E}$ Data Analysis 55(1), 530-543.

Hoff, P. D. (2016). Equivariant and scale-free Tucker decomposition models. Bayesian Anal. 11 (3), 627-648. 
Hoff, P. D., A. E. Raftery, and M. S. Handcock (2002). Latent space approaches to social network analysis. J. Amer. Statist. Assoc. 97(460), 1090-1098.

Holland, P. and S. Leinhardt (1981). An exponential family of probability distributions for directed graphs. Journal of the American Statistical Association 76(373), 33-50.

Hunter, D. R. and M. S. Handcock (2006). Inference in curved exponential family models for networks. J. Comput. Graph. Statist. 15(3), 565-583.

Isard, W. (1954). Location theory and trade theory: short-run analysis. The Quarterly Journal of Economics 68(2), 305-320.

Li, H. and E. Loken (2002). A unified theory of statistical analysis and inference for variance component models for dyadic data. Statist. Sinica 12(2), 519-535.

Lindsay, B. G. (1988). Composite likelihood methods. In Statistical inference from stochastic processes (Ithaca, NY, 1987), Volume 80 of Contemp. Math., pp. 221-239. Amer. Math. Soc., Providence, RI.

Nowicki, K. and T. A. B. Snijders (2001). Estimation and prediction for stochastic blockstructures. J. Amer. Statist. Assoc. 96(455), 1077-1087.

Perry, P. O. (2017). Fast moment-based estimation for hierarchical models. J. R. Stat. Soc. Ser. B. Stat. Methodol. 79(1), 267-291.

Pinheiro, J. C. and D. M. Bates (2000). Mixed-effects models in S and S-PLUS. Berlin; New York: Springer-Verlag Inc.

Price, D. d. S. (1976). A general theory of bibliometric and other cumulative advantage processes. Journal of the Association for Information Science and Technology 27(5), 292-306.

Rohe, K., S. Chatterjee, and B. Yu (2011). Spectral clustering and the high-dimensional stochastic blockmodel. The Annals of Statistics 39(4), 1878-1915.

Snijders, T. A., P. E. Pattison, G. L. Robins, and M. S. Handcock (2006). New specifications for exponential random graph models. Sociological methodology 36(1), 99-153. 
van Duijn, M. A. J., T. A. B. Snijders, and B. J. H. Zijlstra (2004). $p_{2}$ : a random effects model with covariates for directed graphs. Statist. Neerlandica 58(2), 234-254.

Walker, S. C., B. M. Bolker, M. Mchler, and D. Bates (2015). Fitting linear mixed-effects models using lme4. Journal of Statistical Software 67(1), 1-48.

Warner, R., D. A. Kenny, and M. Stoto (1979). A new round robin analysis of variance for social interaction data. Journal of Personality and Social Psychology 37, 1742-1757.

Wasserman, S. and K. Faust (1994). Social Network Analysis: Methods and Applications. Cambridge: Cambridge University Press.

Wasserman, S. and P. Pattison (1996). Logit models and logistic regressions for social networks: I. an introduction to Markov graphs and $p^{*}$. Psychometrika 61(3), 401-425.

Wong, G. Y. (1982). Round robin analysis of variance via maximum likelihood. J. Amer. Statist. Assoc. 777(380), 714-724. 